\begin{tabular}{|c|l|}
\hline Title & Realization of InA s based two-dimensional artificial lattice by sel ective area metal organic vapor phase epitaxy \\
\hline Author(s) & Mohan, Premila; Motohisa, Junichi; Fukui, Takashi \\
\hline Citation & $\begin{array}{l}\text { A pplied Physics Letters, 84(14), 2664.2666 } \\
\text { https://doi.org/10.1063/1697645 }\end{array}$ \\
\hline Issue Date & 20040405 \\
\hline Doc URL & http://hdl.handle.net/2115/5508 \\
\hline Rights & Copyright $\odot 2004$ A merican Institute of Physics \\
\hline Type & article \\
\hline File Information & APL84 14.pdf \\
\hline
\end{tabular}

Instructions for use 


\title{
Realization of InAs-based two-dimensional artificial lattice by selective area metalorganic vapor phase epitaxy
}

\author{
Premila Mohan, ${ }^{\text {a) }}$ Junichi Motohisa, and Takashi Fukui \\ Research Center for Integrated Quantum Electronics (RCIQE), Hokkaido University, \\ North 13 West 8, Sapporo 060-8628, Japan
}

(Received 13 November 2003; accepted 11 February 2004)

\begin{abstract}
The experimental realization of two-dimensional semiconductor artificial lattice based on InAs quantum wires is reported here. Artificial Kagome lattice fabricated using InAs quantum wires of unit cell size $0.7 \mu \mathrm{m}$ has been theoretically proved to show ferromagnetism. Fabrication of such a structure with InAs quantum wires was attempted by selective area metalorganic vapor phase epitaxy using GaAs (111)A substrates. Temperature-dependent growth mode change was observed and Volmer-Weber growth mode at high temperature inhibited the formation of uniform structure. Low temperature and low $\mathrm{AsH}_{3}$ partial pressure resulted in the successful fabrication of $0.7 \mu \mathrm{m}$ period InAs-based Kagome lattice structure. () 2004 American Institute of Physics.
\end{abstract}

[DOI: $10.1063 / 1.1697645$ ]

Recently, much interest has been generated on quantum dots since they are expected to form the basis of nextgeneration high performance devices. The atom-like properties of the quantum dots have made it possible to envision them as building blocks for nanostructures such as artificial molecules and artificial lattice. Such an artificial lattice can be created without the limitations of geometry, composition, and structural stability and may constitute a whole new generation of artificial materials with completely new material properties. This flexibility in the design has led to various theoretical proposals of artificial lattice patterns with possible interesting effects which might not be realized in real materials. One such artificial lattice proposed is a Kagome lattice which is the line graph of the hexagonal lattice. ${ }^{1}$ The remarkable aspect is that the single particle energy spectra have dispersionless flatbands giving rise to flatband ferromagnetism. Successful fabrication of the semiconductor artificial Kagome lattice and the realization of ferromagnetism in such a nonmagnetic semiconductor material will be an important technological breakthrough. The structural features of this nanoscale Kagome lattice will make a good prototype for a wide range of related structures and will open up research in previously inconceivable areas upon which new directions will emerge.

Even though Kagome lattice has been the subject of much theoretical study, further developments depend on the experimental realization which is a technologically challenging task. The principal problem being the atomic or nearatomic control of the placement of nanostructures and equally complicated is the ability to probe their structural and electronic properties. Considering the need and the underlying significance, experimental realization of the artificial semiconductor Kagome lattice structure was undertaken in the present work. The fabrication was based on the theoretical design proposed by Shiraishi, Tamura, and Takayanagi which consists of a network of quantum wires where electrons are localized at the crosspoints of two

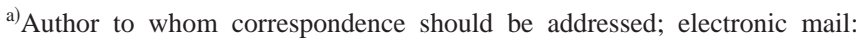
pmohan@rciqe.hokudai.ac.jp
}

wires. ${ }^{2}$ The application of nanoscale fabrication technology and the control and uniformity of nanostructures are vital for the realization of such a complicated structure. Hence, selective area metalorganic vapor phase epitaxy (SA-MOVPE) was chosen, since it is the most promising method for the controlled fabrication of ordered nanostructures without any process induced damage and contamination. Initially, the fabrication was attempted with GaAs quantum wires and 1 $\mu \mathrm{m}$ period GaAs-based Kagome lattice structure was successfully fabricated, ${ }^{3}$ but this pattern period is too large and has to be reduced further to the order of $0.3 \mu \mathrm{m}$ for the realization of ferromagnetism. Unfortunately, design of network structure with such a small period is not feasible with the currently available fabrication techniques, inhibiting further progress with GaAs quantum wires. Eventually, this problem can be overcome by using InAs quantum wires, since it facilitates the possibility of employing a larger pattern period, as the effective mass of InAs is less than that of GaAs. Theoretical calculations reveal that the pattern period required for the experimental realization of ferromagnetism with InAs quantum wire network is $0.7 \mu \mathrm{m}$ period, ${ }^{2}$ the design of which is viable with the current technology.

The fabrication of InAs-based Kagome lattice is not an easy task, since the two-dimensional (2D) InAs heteroepitaxial layer growth is itself difficult, as there is no highly resistive compound semiconductor whose lattice constant

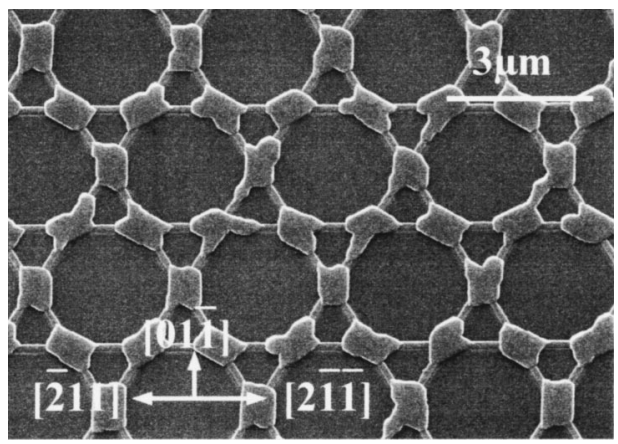

FIG. 1. SEM image of SA-MOVPE grown $3 \mu \mathrm{m}$ period InAs Kagome lattice structure. 

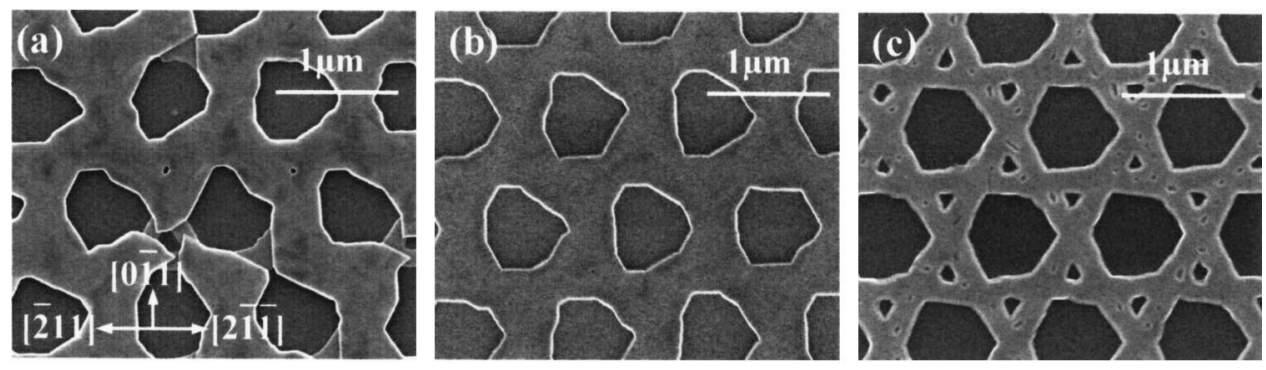

FIG. 2. SEM images of $1 \mu \mathrm{m}$ period Kagome lattice structure grown at (a) $500{ }^{\circ} \mathrm{C}$, (b) $450{ }^{\circ} \mathrm{C}$, and (c) $400{ }^{\circ} \mathrm{C}$. can match that of InAs as a substrate thereby requiring a highly mismatched heteroepitaxy such as the InAs/GaAs system. Due to the large lattice mismatch $(\sim 7.2 \%)$, InAs growth on conventional GaAs (001) substrate usually results in the formation of three-dimensional islands via StranskiKrastanow (SK) growth mode. Recently, it has been reported that the growth mode in the InAs/GaAs system depends on the crystallographic orientation of the substrate surface and 2D growth of InAs could be achieved by using non-(001) substrates. ${ }^{4}$ Such a two-dimensional growth of InAs films on GaAs (111)A substrate by molecular beam epitaxy (MBE) has been reported. ${ }^{5}$ Hence, in the present work, as a first step towards the realization of InAs-based Kagome lattice, 2D growth of InAs on planar GaAs (111)A substrates was attempted by MOVPE.

The growth was carried out using a low-pressure horizontal MOVPE system and the working pressure was kept constant at 76 Torr. Source materials were trimethylgallium (TMGa), trimethyaluminum (TMAl) and 20\% arsine $\left(\mathrm{AsH}_{3}\right)$ in $\mathrm{H}_{2}$. The partial pressures of $\mathrm{AsH}_{3}$ and $\mathrm{TMGa}$ for GaAs buffer layer were $3 \times 10^{-4}$ and $1.6 \times 10^{-6}$ atm, respectively, and that of $\mathrm{AsH}_{3}$ and TMIn for InAs layer were $6.7 \times 10^{-4}$ and $4.2 \times 10^{-7}$ atm, respectively. The growth temperatures of $\mathrm{GaAs}$ and InAs layers were $550^{\circ}$ and $500^{\circ} \mathrm{C}$, respectively. Under these conditions, the growth rates of GaAs and InAs on the planar substrates were 6.67 and $4.76 \mathrm{~nm} / \mathrm{min}$, respectively. The layer structure typically consisted of a $30 \mathrm{~nm}$ GaAs buffer layer followed by a $50 \mathrm{~nm}$ InAs epilayer.

The surface of the as-grown samples was observed using scanning electron microscope (SEM) and atomic force microscope (AFM) and the studies confirmed planar InAs growth with no SK island formation despite the large lattice mismatch. The surface was atomically flat and the rootmean-square (rms) surface roughness determined using AFM was found to be less than $0.7 \mathrm{~nm}$. The results thus indicate that the InAs growth on GaAs (111)A substrates by MOVPE is analogous to that obtained using MBE. ${ }^{5}$ No transformation in the manner of growth, from layer-by-layer to StranskiKrastanow mode, confirms the fact that the misfit strain is released by the formation of misfit dislocations at the InAs/
GaAs interface resulting in the growth of atomically uniform InAs layer. Electrical studies showed $n$-type carrier conduction indicating the presence of donor type impurities or defects and the typical values of the sheet carrier concentration and electron mobility at $77 \mathrm{~K}$ were $1.9 \times 10^{12} \mathrm{~cm}^{-2}$ and $1800 \mathrm{~cm}^{2} / \mathrm{Vs}$, respectively. The grown InAs layers were found to be conducting unlike other semiconductors where carrier depletion occurs for thin layers. This is because, in other semiconductors the surface Fermi level pinning is in the band gap whereas in InAs the surface Fermi level is pinned in the conduction band resulting in native electron accumulation in the near surface region. ${ }^{6}$

After confirming the 2D InAs growth on planar GaAs (111)A substrates, selective area MOVPE growth on patterned substrates was attempted. The mask patterns were formed on 30-nm- $\mathrm{SiO}_{2}$ coated $\mathrm{GaAs}$ (111)A by electron beam lithography and wet chemical etching. SA-MOVPE growth was carried out under the same growth conditions as that employed for planar substrates. Figure 1 shows the SEM image of $3 \mu \mathrm{m}$ period InAs Kagome lattice structure. The grown InAs layer was not continuous and near-island growth occurred, and with no distinct sidewall facets. Growth carried out with still shorter period structures, such as 1.5 and 1 $\mu \mathrm{m}$, indicated that the structure worsened further as the pattern period decreased. In order to improve the structure, growth was carried out at still lower temperature. Figure 2 shows $1 \mu \mathrm{m}$ period Kagome lattice structure grown at $500^{\circ}$, $450^{\circ}$, and $400^{\circ} \mathrm{C}$, respectively. Growth temperature was found to have a strong influence on the growth mode and growth at low temperature resulted in almost perfect Kagome lattice structure with sidewall facets and smooth edges.

This growth mechanism results due to a complex convergence of strain related thermodynamics and growth kinetic effects. Apart from this, the presence of mask was found to influence the growth mechanism significantly. At higher temperature, on patterned substrates, growth proceeds via Volmer-Weber mode (VW), i.e., nucleation and growth of
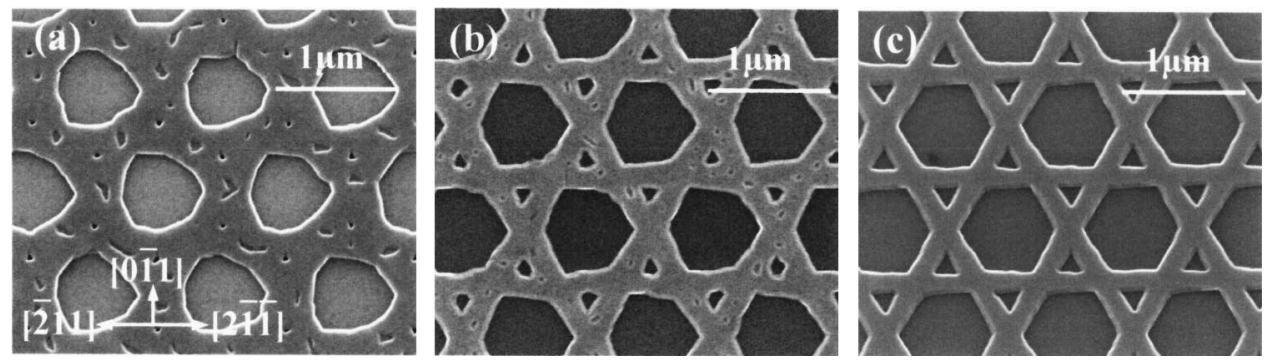

FIG. 3. SEM images of $1 \mu \mathrm{m}$ period Kagome lattice structure grown at $400{ }^{\circ} \mathrm{C}$ with $\mathrm{AsH}_{3}$ partial pressure of (a) $1.67 \times 10^{-3} \mathrm{~atm}$, (b) $6.67 \times 10^{-4}$ atm, and (c) $2.67 \times 10^{-4} \mathrm{~atm}$. 
3D islands, which later coalesce to form a continuous planar InAs layer. The shape of the mask pattern has a strong effect on the nucleation sites as well as the growth of the islands. In the present case, the cross points of two wires act as preferential nucleation sites and at high temperature, the surface migration of In adatom is more, facilitating the adatoms to reach the preferential nucleation sites. Growth proceeds in both vertical as well as lateral directions but, since the threedimensional islands are farther apart, the lateral growth is not sufficient thereby preventing complete coalescence and resulting in near-island growth. Inhomogeneous broadening of islands takes place which results in the complete disappearance of smaller triangular mask area and rough mask edges around the hexagonal mask area. Likewise at $450^{\circ} \mathrm{C}$, the growth proceeds via VW mode but the surface migration distance being comparatively less, facilitates more nucleation sites in addition to the crosspoints of the wires. The increased nucleation sites aid the complete coalescence of the $3 \mathrm{D}$ islands resulting in uniform InAs layer. However, even in this case, the desired Kagome lattice structure was not obtained as the grown InAs layer did not follow the mask pattern. When the growth temperature was further reduced to $400{ }^{\circ} \mathrm{C}$, the average surface migration distance of the adatom becomes lesser, resulting in high-density nucleation sites which in-turn results in uniform layer-by-layer growth via Frank-van der Merwe growth mode. In spite of the large lattice mismatch, layer-by-layer growth mode occurs probably due to the release of misfit strain by the formation of dislocation network at the interface. ${ }^{5}$ This layer-by-layer growth also assists the formation of distinct sidewall facets that arises due to different growth rates on different crystallographic planes.

Another important observation was that the surface morphology deteriorated with decrease in temperature. Even though almost perfect Kagome lattice structure was obtained at $400^{\circ} \mathrm{C}$, the surface morphology was not smooth, with pits appearing on the surface. In order to obtain a smooth surface morphology free of pits, further experiments were carried out at $400{ }^{\circ} \mathrm{C}$ with different $\mathrm{AsH}_{3}$ partial pressure. Figure 3 shows the effect of $\mathrm{AsH}_{3}$ partial pressure on $1 \mu \mathrm{m}$ period Kagome lattice structure. With increase in $\mathrm{AsH}_{3}$ partial pressure, the density of pits was found to increase, and apart from this lateral overgrowth (LOG) on the mask area also occurred resulting in shape deterioration [Fig. 3(a)]. At low $\mathrm{AsH}_{3}$ partial pressure, uniform Kagome lattice structure with atomically flat surface devoid of pits was obtained [Fig. $3(\mathrm{c})]$.

These results originate due to the crystallographic orientation dependence of adatom attachment and can be explained as follows. In the case of GaAs (111)A, the top surface is $\mathrm{Ga}$ terminated in which each surface $\mathrm{Ga}$ atom has one empty dangling bond oriented along the surface normal. The As sites on the (111)A are bound to the underlying $\mathrm{Ga}$ atom by only one bond. These As sites on (111)A thus provide weaker adsorption sites causing the desorption rate to be higher. Hence, high $\mathrm{AsH}_{3}$ partial pressure is required to maintain the continuous growth on (111)A surface but this high $\mathrm{AsH}_{3}$ partial pressure also induces LOG since the group III species can be strongly adsorbed on the $\{110\}$ plane. This is because the $\{110\}$ plane contains both In and As atoms,

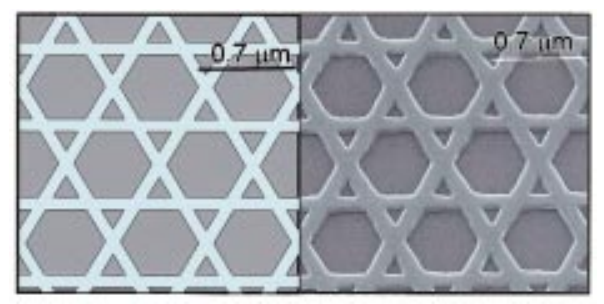

FIG. 4. (Color) (a) Theoretical design of $0.7 \mu \mathrm{m}$ period InAs Kagome lattice structure proposed by Shiraishi, Tamura, and Takayanagi (Ref. 2); (b) experimental design realized by SA-MOVPE.

and the adsorbed As atoms which are bonded to the In atoms, provide additional dangling bonds for the attachment of the In species giving rise to LOG. This probability of In attachment depends on the density of dangling bonds which in turn is related to the availability of As and hence under high As coverage LOG is more pronounced. Due to LOG, the mask pattern assumed a threefold symmetric shape. This is because the $\langle 2-1-1\rangle$ A corners and $\langle-211\rangle$ B corners are crystallographically different and step flow growth occurs only in the $\langle 2-1-1\rangle$ A corners whereas $\{111\} \mathrm{B}$ facet formation in the B corners prevents step flow growth and hence LOG. ${ }^{7}$ Furthermore, the appearance of pits at high $\mathrm{AsH}_{3}$ partial pressure can be attributed to the insufficient adatom migration on the surface. Hence, suppression of LOG at $\langle 2-1-1\rangle$ corners and enhancement of adatom migration are the essential conditions to obtain uniform Kagome lattice structure which could be achieved by employing low $\mathrm{AsH}_{3}$ partial pressure. Experiments revealed that at an $\mathrm{AsH}_{3}$ partial pressure of 2.67 $\times 10^{-4} \mathrm{~atm}, \mathrm{LOG}$ was almost reduced and the top (111) surface was also free of pits. Such an improvement in the surface morphology is due to the enhanced migration of In species at low $\mathrm{AsH}_{3}$ partial pressure. Thus, low growth temperature $\left(400{ }^{\circ} \mathrm{C}\right)$ and low $\mathrm{AsH}_{3}$ partial pressure (2.67 $\times 10^{-4}$ atm) are the optimum conditions to obtain uniform Kagome lattice structure with shorter pattern period. The pattern period was further reduced and selective area growth carried out using these optimized conditions resulted in the successful fabrication of $0.7 \mu \mathrm{m}$ period InAs Kagome lattice structure (Fig. 4).

In summary, we have experimentally realized the theoretically proposed $0.7 \mu \mathrm{m}$ InAs-based Kagome lattice by employing selective area MOVPE. Such a structure is expected to show flatband ferromagnetism and semiconductor ferromagnets can be realized in the future with this structure as the basis. Apart from this significant technological application, it can also serve as the perfect candidate for studying the physics of strongly correlated manybody systems.

${ }^{1}$ A. Mielke, J. Phys. A 24, L73 (1991).

${ }^{2}$ K. Shiraishi, H. Tamura, and H. Takayanagi, Appl. Phys. Lett. 78, 3702 (2002).

${ }^{3}$ P. Mohan, F. Nakajima, M. Akabori, J. Motohisa, and T. Fukui, Appl. Phys. Lett. 83, 689 (2003).

${ }^{4}$ X. M. Zhang, D. W. Pashley, I. Kamiya, J. H. Neave, and B. A. Joyce, J. Cryst. Growth 147, 234 (1995).

${ }^{5}$ H. Yamaguchi, J. G. Belk, X. M. Zhang, J. L. Sudijono, M. R. Fahy, T. S. Jones, D. W. Pashley, and B. A. Joyce, Phys. Rev. B 55, 1337 (1997).

${ }^{6}$ H. Yamaguchi, R. Dreyfus, Y. Hirayama, and S. Miyashita, Appl. Phys. Lett. 78, 2372 (2001).

${ }^{7}$ M. Akabori, J. Motohisa, and T. Fukui, in Proceedings of the 27th International IEEE Conference on Compound Semiconductors (2001), p. 191. 